\title{
Absceso del psoas ilíaco secundario a un tumor de colon izquierdo fistulizado al retroperitoneo. Caso clínico y análisis de la literatura.
}

Iliopsoas abscess resulting from penetrating left colon cancer to the retroperitoneum.

Case report and literature review.

Abscesso do iliopsoas secundário a tumor de cólon esquerdo fistulizado para o retroperitônio.

Caso clínico e análise da literatura.

Alejandra Liz ${ }^{1}$

ORCID 0000-0002-1530-4782

alejandraliz@montevideo.com.uy

Emilia Cerchiari $^{2}$

ORCID 0000-0002-4619-829X

emilia.cerchiari@gmail.com

\author{
Gonzalo Gayo ${ }^{3}$ \\ ORCID: 0000-0002-3931-6403 \\ ggayo@adinet.com.uy
}

Diego Lapiedra ${ }^{4}$

ORCID 0000-0002-1209-1697

diegolapiedra@hotmail.com

Marcelo Viola ${ }^{5}$

ORCID 0000-0003-2733-5276

mviolam@gmail.com

Marcelo Diamant ${ }^{6}$

ORCID 0000-0001-9708-6688

diamantm21@gmail.com

DOI 10.31837/cir.urug/6.1.7

Recibido: 5 de abril de 2021

Aceptado: 6 de febrero de 2022

\section{Resumen}

Los abscesos del psoas ilíaco secundarios a un tumor de colon fistulizado son excepcionales y potencialmente graves. La mayoría son adenocarcinomas de tipo mucinoso. Su tratamiento es complejo ya que, para lograr una resección oncológica pretendidamente curativa, es necesario realizar una resección ampliada con mayor morbimortalidad. Presentamos el caso de una paciente con un adenocarcinoma mucinoso de colon izquierdo fistulizado al músculo ilíaco y la pared anterolateral del abdomen en la que se realizó una resección multivisceral que incluyó el colon izquierdo, el músculo y la cresta ilíaca y parte de la pared anterolateral del abdomen.

Palabras clave: cáncer de colon, absceso del psoas, fistulización, perforación, adenocarcinoma mucinoso.

\footnotetext{
${ }^{1}$ Jefe de Residentes, Clínica Quirúrgica “1”, Prof. Dr. Fernando González. Hosp. Pasteur, Fac. de Medicina, UdelaR, Montevideo, Uruguay

${ }^{2}$ Asistente de clase, Clínica Quirúrgica “1”, Prof. Dr. Fernando González. Hosp. Pasteur, Fac. de Medicina, UdelaR, Montevideo, Uruguay

${ }^{3}$ Prof. Adjunto, Clínica Quirúrgica "1”, Prof. Dr. Fernando González. Hosp. Pasteur, Fac. de Medicina, UdelaR, Montevideo, Uruguay

${ }^{4}$ Prof. Adjunto, Clínica Quirúrgica “1”, Prof. Dr. Fernando González Hosp. Pasteur, Fac. de Medicina, UdelaR, Montevideo, Uruguay

${ }^{5}$ Prof.Agregado, Clínica Quirúrgica “1”, Prof. Dr. Fernando González. Hosp. Pasteur, Fac. de Medicina, UdelaR, Montevideo, Uruguay

${ }^{6}$ Prof. Agregado, Clínica Quirúrgica “1”, Prof. Dr. Fernando González. Hosp. Pasteur, Fac. de Medicina, UdelaR, Montevideo, Uruguay
} 


\begin{abstract}
Iliopsoas abscess secondary to perforation of colon cancer is an extremely rare and potentially lifethreatening condition. Most tumors are mucinous adenocarcinomas. Its treatment its complex, as most patients need radical extended resections to achieve good oncological results, which are in turn, graved with higher morbidity and mortality. We present the case of a patient with a left colon mucinous adenocarcinoma penetrating to the iliopsoas muscle and the anterolateral abdominal wall that required a multivisceral resection including left colon, iliac muscle and crest and part of the anterolateral abdominal wall.
\end{abstract}

Keywords: colon cancer, psoas abscess, fistulization, perforation, mucinous adenocarcinoma.

\title{
Resumo
}

Abscessos do iliopsoas secundários a um tumor de cólon fistulizado são raros e potencialmente graves. A maioria são adenocarcinomas do tipo mucinoso. Seu tratamento é complexo, pois, para se obter uma ressecção oncológica supostamente curativa, é necessário realizar uma ressecção ampliada com maior morbimortalidade. Apresentamos o caso de um paciente com adenocarcinoma mucinoso de cólon esquerdo fistulizado para o músculo ilíaco e parede ântero-lateral do abdome no qual foi realizada ressecção multivisceral que incluiu cólon esquerdo, músculo e crista ilíaca e parte do a parede anterolateral do abdome.

Palavras chave: câncer de cólon, abscesso do psoas, fistulização, perfuração, adenocarcinoma mucinoso.

\section{Introducción}

Las infecciones retroperitoneales han preocupado a la comunidad quirúrgica durante décadas debido a la dificultad en el diagnóstico y a su considerable mortalidad, estimada en un $20 \%$. En el pasado, las causas más comunes eran los denominados abscesos "fríos" secundarios a tuberculosis. Actualmente, los más frecuentes son los considerados "calientes", debidos a enfermedades inflamatorias o infecciosas intraabdominales o retroperitoneales. Es excepcional que un absceso retroperitoneal sea secundario a una perforación de un cáncer de colon. En estos casos, la presentación clínica es particularmente atípica.

El retraso diagnóstico y el manejo inadecuado representan la principal causa de aumento de morbimortalidad en este particular grupo de pacientes.

Comentamos el caso clínico de una paciente que se presentó con un absceso del psoas ilíaco por fistulización de un tumor de colon izquierdo. 


\section{Caso clínico}

Se trata de una mujer de 53 años que consultó por dolor en fosa ilíaca izquierda y dificultad en la marcha. La tomografía computada (TC) abdominopélvica evidenció un absceso del músculo ilíaco izquierdo y un engrosamiento del colon izquierdo.

Se realizó el drenaje percutáneo de la colección, obteniendo escaso líquido mucinoso.

En la evolución, desarrolló una obstrucción intestinal que requirió una colostomía en asa transversa de detransitación, al comprobarse un tumor de colon descendente que infiltraba ampliamente los vasos ilíacos y el músculo psoas ilíaco izquierdo.

Se completó la valoración con una video-colonoscopía que evidenció, sobre la unión descendento-sigmoidea, una lesión de aspecto velloso con abundante moco, cuya biopsia mostró un adenoma con displasia de alto grado.

Una nueva TC de estadificación demostró la infiltración del tumor al músculo ilíaco y nervio crural izquierdo. La arteria ilíaca externa se encontraba en íntimo contacto sin invasión aparente. Se objetivó una colección sobre la pared abdominal con relación a la cicatriz mediana (Fig. 1). No había evidencia de diseminación a distancia. Los marcadores tumorales CEA y CA 19-9 fueron normales.

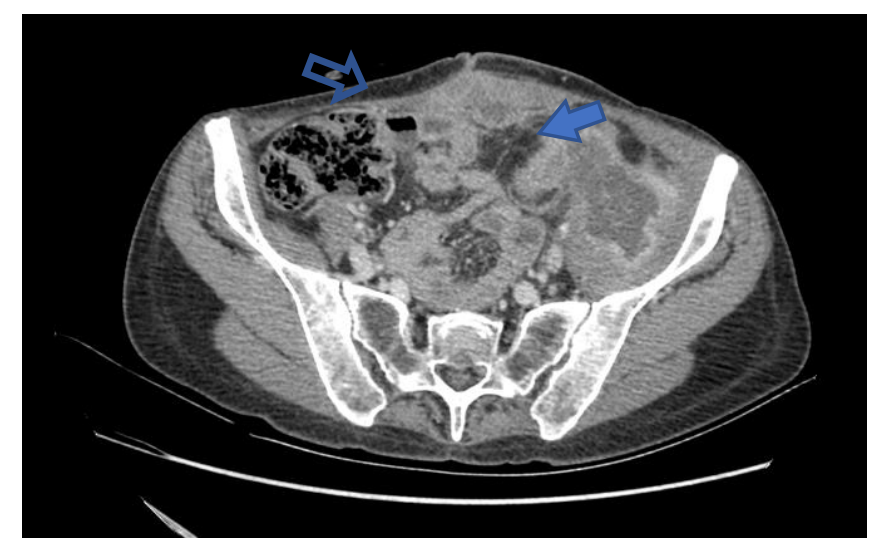

Fig. 1. a) Engrosamiento del colon descendente con fistulización hacia el músculo ilíaco izquierdo y colección en su interior (flecha sólida). b) Colección detrás de la mediana (flecha vacía).

Durante este período, la paciente agregó supuración sobre la cicatriz y una alteración inflamatoria sobre la fosa ilíaca izquierda (Fig. 2).

El diagnóstico preoperatorio fue un cáncer de colon izquierdo fistulizado al músculo psoas ilíaco izquierdo y a la pared abdominal. Se discutió en ateneo multidisciplinario en conjunto con equipos de oncología, cirugía general, vascular, ortopedia y reparadora, decidiendo realizar una resección multivisceral en bloque con criterio curativo intentando una resección R0. 


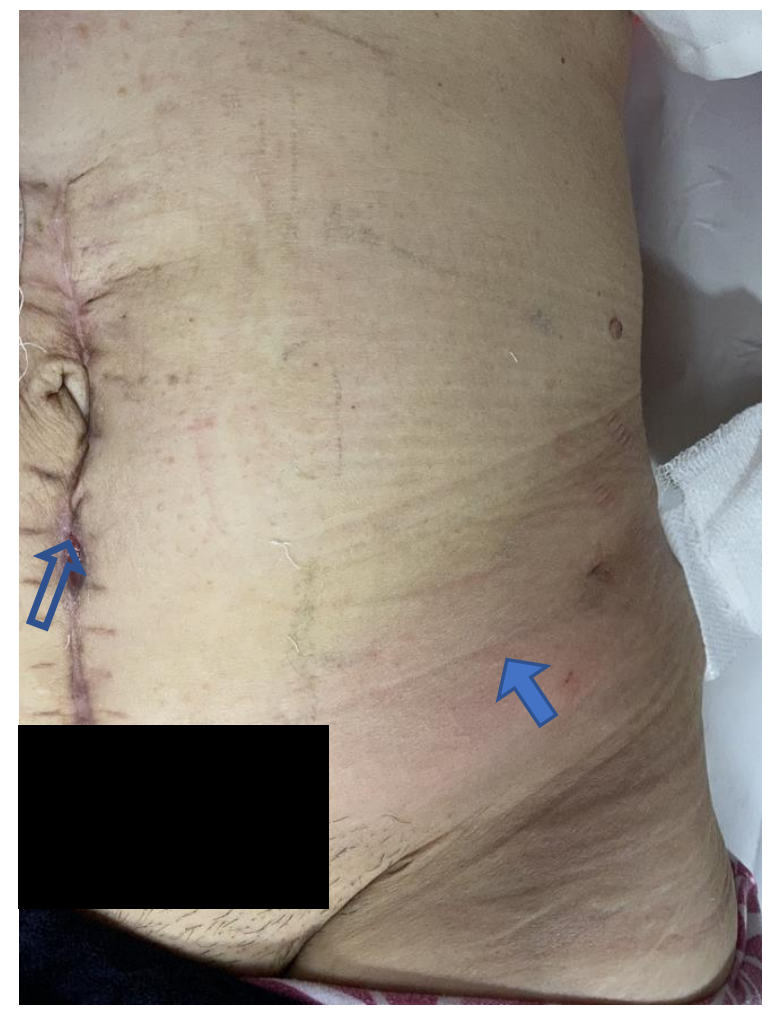

Fig. 2. a) Área inflamatoria por compromiso cutáneo del tumor (flecha sólida). b) Orificio fistuloso de la cicatriz mediana (flecha vacía).

El diagnóstico preoperatorio fue de cáncer de colon izquierdo fistulizado al músculo ilíaco izquierdo y a la pared abdominal. Se conformó un equipo multidisciplinario con servicios de oncología, cirugía general, vascular, ortopedia y reparadora, decidiendo realizar una RMV con criterio curativo. La cirugía consistió en una colectomía izquierda ensanchada con resección en bloque del músculo ilíaco, nervio crural, cresta ilíaca y pared anterolateral del abdomen (Fig. 3).

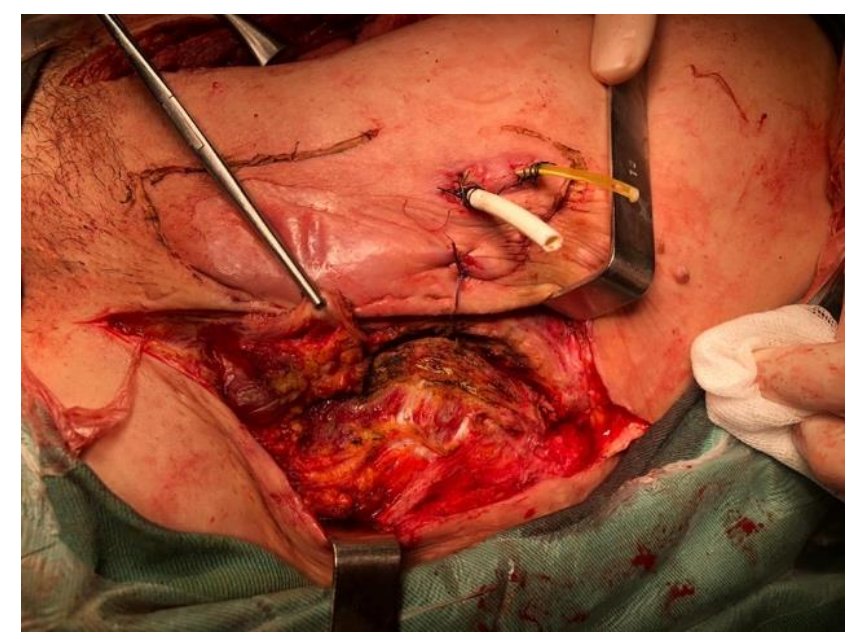

Fig. 3. Resección en bloque incluyendo el área de drenaje percutáneo del absceso (la cabeza de la paciente se encuentra hacia la derecha). 
La anatomía patológica informó un carcinoma mucinoso pT3N0 (0+/14) márgenes libres, R0. En un segundo tiempo, se realizó la cobertura de la pared abdominal faltante con colgajo anterolateral de muslo derecho. La paciente tuvo una excelente evolución sin complicaciones mayores ni secuelas funcionales graves, quedando con una cojera de la pierna izquierda que soluciona con bastón. Sin recaída, a 11 meses de la cirugía.

\section{Discusión}

Los abscesos retroperitoneales son patologías potencialmente graves, con una elevada morbimortalidad a menos que sean diagnosticados y tratados precozmente. Esto se debe a que el espacio retroperitoneal no reacciona en forma aguda a la infección a diferencia de la cavidad peritoneal. Cuando son debidos a una perforación del tracto gastrointestinal, las manifestaciones clínicas extraperitoneales son particularmente engañosas.

El primer absceso del psoas fue descrito por Mynter en 1881 con el nombre de "psoitis". ${ }^{(1)} \mathrm{Su}$ incidencia se estima en 0.5 a 6.5 casos por 10000 ingresos hospitalarios. Clásicamente, los abscesos del psoas pueden dividirse en primarios o secundarios, dependiendo de la presencia o no de una patología subyacente. ${ }^{(2)}$ En una revisión de 367 casos, Ricci et al. evidenciaron que la etiología variaba según la geografía. En Asia y África, los abscesos primarios del psoas comprenden más del 90\% de los casos, siendo menos frecuentes en otras partes del mundo (20$30 \%$ ) como Europa y Norteamérica, donde el $70 \%$ corresponden a abscesos secundarios. ${ }^{(2)}$ Los abscesos primarios ocurren por diseminación hematógena, habitualmente desde un foco oculto y predominan en pacientes inmunocomprometidos. Los abscesos secundarios resultan de la extensión local de un proceso infeccioso vecino. La enfermedad de Crohn es la causa más frecuente con el $60 \%$ de los casos. Le siguen en frecuencia la apendicitis en $16 \%$ y la colitis ulcerosa crónica y la diverticulitis en $11 \%$. ${ }^{(3)}$

Los abscesos del psoas debido a perforación de cáncer de colon son extremadamente raros, siendo esta la primera comunicación nacional de esta complicación. Su incidencia estimada es del 0.3 y $0.4 \%$, mientras que la de la perforación libre es 3 a 10\%. Se observan principalmente en el colon izquierdo. En una serie de 501 pacientes con abscesos intraabdominales, el 3\% correspondieron a tumores colónicos perforados. La formación de una fístula es menos frecuente, observándose en el 15\% de todas las perforaciones colónicas, sin predilección por algún sector de colon en particular. ${ }^{(4)}$ Tsai et al. analizaron retrospectivamente 756 casos de cáncer de colon durante 6 años e identificaron solamente 2 casos $(0.26 \%)$ de abscesos intraabdominales como forma de presentación del cáncer de colon. Esta complicación tiene una mortalidad de $50 \%$ y la sobrevida a 5 años es del $20 \%$. $^{(5)}$

Michowitz et al. propusieron una clasificación de complicaciones perforativas por cáncer de colon que pueden dividirse en: perforación en cavidad libre con peritonitis; perforación con formación de abscesos y perforación a un órgano vecino o formación de una fístula. ${ }^{\left({ }^{6}\right)} \mathrm{La}$ perforación y penetración a órganos adyacentes puede explicarse por necrosis de úlceras estercoráceas o hipertensión proximal de una lesión obstructiva. 
El absceso puede localizarse en el área paracólica, extenderse como un absceso de flanco, dirigirse hacia el espacio retrorrenal y del psoas desarrollando un absceso lumbar o hacia la fosa ilíaca con la formación de un absceso ilíaco e incluso, atravesar varios planos tisulares y presentarse como un absceso de otra topografía como el muslo o el espacio subcutáneo del tronco. ${ }^{(7)}$

En la patogenia de los abscesos retroperitoneales por perforación tumoral, debe considerarse al adenocarcinoma mucinoso. Estos son considerados tumores raros. Suelen tener crecimiento lento y habitualmente se diseminan por extensión directa. Se ha reportado una mayor incidencia de carcinomas mucinosos entre los tumores de colon que se presentan como abcesos de la pared abdominal. ${ }^{(3)}$ En esta variante, se observa que la actividad enzimática de la arilsulfatasa y las lisozimas es más elevada que en otros tipos de adenocarcinomas. Estas enzimas disminuyen el grado de sulfatación del heparán sulfato y degradan la barrera de proteoglicanos. Cuando la barrera se destruye, el adenocarcinoma mucinoso aumenta su habilidad para infiltrar. Las células tumorales infiltran la pared intestinal formando fístulas o llevando al desarrollo de abscesos en los tejidos infiltrados. ${ }^{(7)}$

La variedad mucinosa está presente en la mayoría de los reportes de abscesos retroperitoneales secundarios a cáncer de colon. Mohandas et al. reportaron un tumor de ciego fistulizado a la pared posterolateral del abdomen y grasa subcutánea de la región glútea. La anatomía patológica mostró un adenocarcinoma mucinoso de colon. ${ }^{(8)}$ Wang et al. publicaron un absceso del psoas derecho secundario a un adenocarcinoma mucinoso. ${ }^{(7)}$ Estos hallazgos coinciden con el caso clínico que presentamos.

La elevada mortalidad de las infecciones retroperitoneales se debe, en parte, a las dificultades en el diagnóstico. Las perforaciones colónicas retroperitoneales causantes de abscesos se presentan como un grupo heterogéneo de signos y síntomas por lo que su diagnóstico puede ser difícil. Dos signos orientan a la perforación retroperitoneal: la presencia de gas extraintestinal y el absceso del músculo psoas ilíaco. ${ }^{(9)}$ La clásica tríada de fiebre, dolor abdominal o dorsal y cojera fue reportada por Mallick et al. ${ }^{(3)}$ en $30 \%$ de los pacientes y en $5 \%$ para Dietrich et al. (3) El diagnóstico de absceso del psoas puede retrasarse debido a que otras enfermedades inflamatorias pueden presentarse con una clínica similar. Choi describió el mecanismo de diseminación de abscesos, gas y necrosis a lo largo del retroperitoneo y mediastino a través de la conexión anatómica entre las regiones. ${ }^{(9)}$ En 1974, Meyers reportó que el gas puede progresar desde el espacio pararrenal posterior a través del hiato diafragmático causando neumomediastino y enfisema subcutáneo cervical. ${ }^{(9)}$ La TC abdominal y la resonancia nuclear magnética (RNM) son los estudios de elección para la evaluación de los abscesos del psoas. El hallazgo de áreas hipodensas con refuerzo periférico dentro de un psoas aumentado de tamaño, son fuertemente sugestivas de abscesos. ${ }^{(3)}$ La tomografía también permite la estadificación preoperatoria ${ }^{(5)}$ y delinear la comunicación entre el absceso del psoas y el tumor, como sucedió en el caso de nuestra paciente. La realización de una video-colonoscopía identifica la lesión y la biopsia confirma la etiología. Cuando no puede llegarse al diagnóstico por biopsia endoscópica puede ser necesaria la biopsia percutánea o, como en nuestro caso, optar por la cirugía dada la alta sospecha diagnóstica. ${ }^{(7)}$ En ocasiones, el diagnóstico de cáncer se realiza en el postoperatorio debido a que los pacientes se presentan con cuadros clínicos que requieren 
tratamiento urgente. ${ }^{(9)}$ Existen situaciones en las que el tumor solo se evidencia luego de superado el proceso infeccioso ${ }^{(3)} \mathrm{o}$ se sospecha debido a la mala evolución luego del drenaje. ${ }^{(3)}$

El tratamiento del cáncer de colon perforado hacia el retroperitoneo puede ser complejo. Okita reportó que la táctica quirúrgica depende de la estadificación. En ausencia de metástasis a distancia, la resección del absceso y la fístula en bloque con el tumor es el tratamiento de elección. ${ }^{(10)}$

El drenaje exclusivo se utiliza como tratamiento paliativo en pacientes con mal estado general que no toleran procedimientos resectivos. ${ }^{(6)}$

\section{Conclusión}

Los tumores de colon pueden presentar síntomas atípicos como en este caso, que se presentó como un cáncer fistulizado al psoas ilíaco izquierdo. Luego del drenaje percutáneo, se optó por una resección multivisceral en bloque obteniendo una resección R0 y se realizó la reconstrucción de la pared anterolateral del abdomen con un excelente resultado funcional y oncológico.

\section{Bibliografía}

1. Mynter H. Acute psoitis. Buffalo Med Surg J 1881;21:202-210.

2. Ricci MA, Rose FB, Meyer KK. Pyogenic psoas abscess: worldwide variations in etiology. World J Surg 1986;10(5):834-43. doi: 10.1007/BF01655254.

3. Menon A, Agashe VM, Jakkan MS. Rare case of iliopsoas abscess secondary to mucinous adenocarcinoma of the colon: a case report. J Orthop Case Rep 2018;8:32-5. doi: 10.13107/jocr.2250-0685.986.

4. Peterson CM, Allison JG, Lu CC. Psoas abscess resulting from perforating carcinoma of the sigmoid colon: report of a case. Dis Colon Rectum 1983; 26:390-2. doi: 10.1007/BF02553381.

5. Tsai HL, Hsieh JS, Yu FJ, Wu DC, Chen FM, Huang CJ, Huang YS, Huang TJ, et al. Perforated colonic cancer presenting as intra-abdominal abscess. Int J Colorectal Dis 2007;22(1):15-9. doi: 10.1007/s00384-006-0097-6.

6. Cacurri A, Cannata G, Trastulli S, Desiderio J, Mangia A, Adamenko O, et al. A rare case of perforated descending colon cancer complicated with a fistula and abscess of left iliopsoas and ipsilateral obturator muscle. Case Rep Surg 2014;128506: doi: 10.1155/2014/128506.

7. Wang J, Li Honglang, Xiong B, Wu L, Hu J. A case report of ileocecal mucinous adenocarcinoma with abscesses in the low-right abdomen and formation of sinus tract in the abdominal wall. Int J Clin Exp Med 2017;10(9):14001-8. 
8. Mohandas SK, Mazarello F, Bisset R. Right gluteal abscess: an unusual presentation of perforated caecal adenocarcinoma. J Gastrointest Canc 2010; 41:285-7. doi: 10.1007/s12029-010-9155-y

9. Ruscelli P, Renzi C, Polistena A, Sanguinetti A, Avenia N, Popivanov G, et al. Clinical signs of retroperitoneal abscess from colonic perforation. Two case reports and literature review. Medicine 2018; 97:45. e13176.

doi: 10.1097/MD.0000000000013176.

10. Okita A, Kubo Y, Tanada M, Kurita A, Takashima S. Unusual abscesses associated with colon cancer: report of three cases. Acta Med Okayama 2007;67(2):107-13. doi: 10.18926/AMO/32885.

\section{Contribución autores:}

Alejandra Liz: Concepción, diseño, análisis, redacción.

Emilia Cerchiari: Diseño, redacción.

Diego Lapiedra: Análisis.

Gonzalo Gayo: Análisis.

Marcelo Viola: Revisión crítica.

Marcelo Diamant: Revisión crítica.

Los autores declaran que no existe conflicto de interés

Nota: El editor Gustavo Rodríguez Temesio aprobó este artículo 